\title{
DATA PRIVACY IN ENCRYPTED RELATIONAL DATA USING K-NN CLASIFICATION
}

\author{
M.Sheshikala \\ Assistant Professor, CSE Department \\ $\mathrm{S}$ R Engineering College \\ Warangal, India
}

\author{
R. Vijaya Praash \\ Professor, CSE Department \\ S R Engineering College \\ Warangal, India
}

\author{
M.Archana \\ M.Tech, CSE Department \\ S R Engineering College \\ Warangal, India
}

\begin{abstract}
Data Mining has wide applications in various domains, for instance, keeping cash, arrangement, intelligent research and among government workplaces. Portrayal is one of the usually used assignments in data mining applications. As far back as decade, due to the climb of various assurance issues, various theoretical and convenient responses for the request issue have been proposed under different security models. In any case, with the present pervasiveness of circulated processing, customers now have the opportunity to outsource their data, fit as a fiddle, and moreover the data mining endeavors to the cloud. Since the data on the cloud is in encoded outline, existing security protecting plan procedures are not significant. In this paper, we focus on dealing with the gathering issue over encoded data. In particular, we propose a secured k-NN classifier over mixed data in the cloud. The proposed tradition guarantees the characterization of data, security of customer's information question, and covers the data get to outlines. To the best of our knowledge, our work is the first to develop an ensured kNN classifier over encoded data under the semi-authentic model. In like manner, we observationally inspect the profitability of our proposed tradition using a bona fide dataset under different parameter settings.
\end{abstract}

Keywords: Security, k-NN Classfier, Outsourced databases, encryption

\section{INTRODUCTION}

Starting late, the conveyed registering perspective [1] is adjusting the affiliations' strategy for working their data particularly in the way they store, get to and get ready data. As a creating handling perspective, conveyed figuring pulls in various relationship to consider really regarding cloud potential to the extent its cost viability, flexibility, and offload of administrative overhead. Regularly, affiliations choose their computational operations despite their data to the cloud. Notwithstanding tremendous purposes of intrigue that the cloud offers, assurance and security issues in the cloud are suspecting associations to utilize those inclinations. Exactly when data are extremely fragile, the data ought to be encoded before outsourcing to the cloud. Regardless, when data are mixed, paying little respect to the shrouded encryption plot, playing out any data mining assignments ends up being amazingly trying while never unscrambling the data. There are other security concerns, appeared by the going with case. Case 1. Accept a protection organization outsourced its encoded customers database and imperative data mining assignments to a cloud. Right when an administrator from the association needs to choose the risk level of a potential new customer, the expert can use a request method to choose the peril level of the customer. To begin with, the administrator needs to make a data record $\mathrm{q}$ for the customer containing certain individual information of the customer, e.g., money related appraisal, age, matrimonial status, et cetera. By then this record can be sent to the cloud, and the cloud will figure the class stamp for q. Coincidentally, since q contains fragile information, to secure the customer's assurance, q should be mixed before sending it to the cloud. The above case shows that data mining over mixed data (implied by DMED) on a cloud also needs to secure a customer's record when the record is a bit of a data mining process. Moreover, cloud can in like manner induce accommodating and fragile information about the genuine data things by viewing the data get to outlines paying little mind to the likelihood that the data are mixed [2], [3]. Subsequently, the insurance/security essentials of the DMED issue on a cloud are triple: (1) protection of the encoded data, (2) arrangement of a customer's question record, and (3) disguising data get to outlines. Existing work on assurance sparing data mining (PPDM) (either trouble or secure multi-party estimation (SMC) based approach) can't deal with the DMED issue. Irritated data don't have semantic security, so data aggravation systems can't be used to scramble exceedingly delicate data. Moreover the aggravated data don't make to a great degree exact data mining comes to fruition. Secure multi-party computation based approach expect data are scattered and not mixed at each sharing gathering. Moreover, many direct figuring's are performed in perspective of non-mixed data. In like manner, in this paper, we proposed novel strategies to satisfactorily deal with the DMED issue tolerating that the encoded data are outsourced to a cloud. Specifically, we focus on the gathering issue since it is a champion among the most broadly perceived data mining endeavors. Since each portrayal system has their own particular great position, to be strong, this paper concentrates on executing the k-nearest neighbor arrange method over encoded data in the disseminated processing condition. As a making dealing with model, cloud prepare attracts different relationship to consider truly 
concerning cloud potential concerning its cost capacity, adaptability, and offload of association cost. From time to time, affiliations entrust their computational points of confinement in change to their data to the cloud. In spite of radiant inclinations that the cloud offers, security and solace issues in the thinking are staying away from relationship to use those central focuses. Precisely when data is altogether tricky, the data should be encoded before outsourcing to the cloud. Taking everything in account, when data are secured, paying little respect to the true blue security course of action, executing any data mining errands changes into incredibly scrambled while never unscrambling the data. There are other security stresses, bore witness to by the running with test. Test 1: expect a protection supplier gotten its secured customers database and basic information mining errand to a cloud. Precisely when a delegate from the affiliation needs to comprehend the peril time of a potential new customer, the administrator can utilize an approach system to fathom the hazard time of the customer. Starting, the delegate requires conveying a reasons for interest history $\mathrm{q}$ for the customer containing certain private unnoticeable parts of the customer, e.g., FICO examination, age, marriage status, and so forth. By then this history can be sent to the cloud, and the cloud will assess the class stamp for q. In any case, since q contains fragile subtle segments, to secure the client's protection, $\mathrm{q}$ ought to be encoded before passing on it to the cloud. The above case uncovers that information mining over encoded data (inferred by DMED) on a cloud in like way requires securing a client's history when the history is somewhat of an information mining strategy. Also, cloud can in like way get enduring and sensitive data about the true blue data things by viewing the data availability styles paying little personality to the way that the data are encoded [4], [5]. Accordingly, the confirmation/security inconspicuous components of the DMED issue on a cloud are triple: (11) solace of the encoded data, (12) solace of a client's question history, and (13) covering data openness arranges. Current work on security saving information mining (PPDM) (either inconvenience or ensured multi-party estimation (SMC) focused method) can't modify the DMED issue. Irritated data don't have semantic insurance, so data inconvenience procedures can't be related with secure remarkably sensitive data. In like way the pestered data don't convey especially amend data mining comes to fruition. Secure multi-party numbers focused technique addresses data are spread and not secured at each taking including gathering. In thought, different pushed estimations are driven relying on non-encoded data. As needs be, in this paper, we endorsed novel timetables to effectively resolve the DMED issue tolerating that the secured data is contracted to a cloud. Especially, we focus on the class issue considering that it is a champion among the most by and large saw information mining tries. For the reason that each request logic has their own central focuses, to be unmistakable, this record concentrates on playing out the k-closest neighbor portrayal system over secured data in the cloud prepare air. A. System Model and Problem Definition In our issue setting, we consider $\mathrm{n}$ customers implied by U1, . . . , Un. Accept customer Ui holds a database Ti with mi data records and 1 qualities, for $1=\mathrm{i}=\mathrm{n}$. Consider a circumstance where the $\mathrm{n}$ customers need to outsource their databases and furthermore the k-infers gathering process on their merged databases to a cloud space. In our system appear, we consider two unmistakable components: (i) the customers and (ii) the cloud pro centers. We acknowledge that the customers pick two cloud pro associations C1 and C2 (say Amazon and Google) to play out the clustering undertaking on their joined data. In this paper, we unequivocally expect that C1 and C2 are semireasonable [6] and they don't interest. After proper organization level concurrences with the customers, C2 produces an open secret key join (pk, sk) in light of the Paillier cryptosystem [7] and conveys pk to all customers and C1. A more generous setting would be for C1 and C2 to commonly make individuals as a rule enter pk in light of the farthest point Paillier cryptosystem (e.g., [8], [9]) with the true objective that the contrasting secret key sk is thoughtlessly part between the two fogs. Under this case, the secret key sk is dark to both fogs and simply (discretionary) shares of it are revealed to C1 and C2. For straightforwardness, we consider the past uneven setting where C2 makes (pk, sk) in the straggling leftovers of this paper. Regardless, our proposed tradition can be easily contacted as far as possible setting without affecting the essential security guarantees. Given the above system designing, we acknowledge that customer $\mathrm{Ui}$ encodes $\mathrm{Ti}$ property adroit using pk and outsources the mixed database to C1. Another way to deal with outsource the data is that customers can part every trademark impetus in their database into two sporadic shares and outsource the shares freely to each cloud (see Section V-B for more purposes of intrigue).

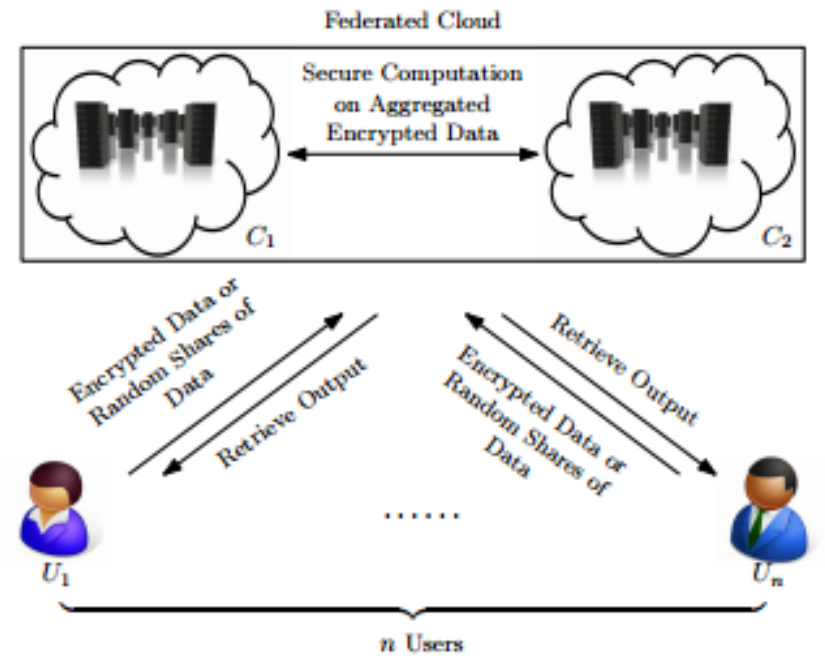

Fig. 1. The Proposed PPODC Architecture

\section{RELATED WORK AND BACKGROUND}

As a result of space hindrances, here we rapidly review the current related work and give a couple of definitions as an establishment. In the event that it's not all that much inconvenience insinuate our particular report [5] for a more clarified related work and establishment. At in any case, it gives off an impression of being totally homomorphic cryptosystems (e.g., [6]) can handle the DMED issue since it allows a third party(that has the encoded data) to execute optional limits over mixed data while never unscrambling them. Regardless, we extend that such strategies are to a great degree exorbitant and their utilization in valuable applications still can't be researched. For example, it was showed up in [7] that despite for weak security parameters one ?bootstrapping? operation of the homomorphic operation would take no under 30 seconds on a world class machine. It is possible to use the present secret sharing methodology in SMC, for instance, 
Shamir's arrangement [8], to develop a PPkNN tradition. Regardless, our work is not the same as the secret sharing based course of action in the going with perspective. Game plans in light of the puzzle sharing arrangements require no under three get-togethers while our work require only two gettogethers. For example, the advancements in perspective of Share cerebrum [9], an exceptional SMC structure which relies on upon the riddle sharing arrangement, acknowledge that the amount of partaking social affairs is three. Along these lines, our work is orthogonal to Share mind and other puzzle sharing based arrangements. 2.1 Privacy-Preserving Data Mining Agrawal and Srikant [10], Lindell and Pinkas [11] were the first to exhibit the prospect of security protecting under data mining applications. The current PPDM techniques can broadly be orchestrated into two arrangements: (i) data disturbance and (ii) data dissemination. Agrawal and Srikant [10] proposed the fundamental data trouble system to make a decision tree classifier, and various diverse methodologies were proposed later (e.g., [12]). In any case, as said earlier in Section 1, data bothering systems can't be pertinent for semantically secure mixed data. In like manner, they don't convey correct data mining happens as a result of the development of accurate hullabaloos to the data. On the other hand, Lindell and Pinkas [11] proposed the essential decision tree classifier under the two party setting tolerating the data were dispersed between them. Starting now and into the foreseeable future much work has been dispersed using SMC strategies (e.g., [12]). We state that the PPkNN issue can't be fathomed using the data scattering systems since the data for our circumstance is mixed and not appropriated in plaintext among various social affairs. For comparative reasons, we in like manner don't consider secure k-NN systems in which the data are passed on between two social events. Query Processing over Encrypted Data diverse systems related to request taking care of over mixed data have been proposed, e.g., [13], [14], [8]. Regardless, we watch that PPkNN is a more unusual issue than the execution of essential $\mathrm{kNN}$ request over encoded data [15], [16]. For one, the widely appealing $\mathrm{k}$ nearest neighbors in the portrayal technique, should not be revealed to the cloud or any customers. We underscore that the present system in [17] reveals the knearest neighbors to the customer. Second, paying little mind to the likelihood that we know the k-nearest neighbors, it is still uncommonly difficult to find the lion's share class stamp among these neighbors since they are encoded at the essential spot to shield the cloud from learning sensitive information. Third, the present work did no watched out for the get to case issue which is a huge assurance need from the customer's perspective. In our most recent work, we proposed a novel secure k-nearest neighbor request tradition over encoded data that guarantees data mystery, customer's question insurance, and hides data get to plans. In any case, as said above, PPkNN is a more personality boggling issue and it can't be lit up clearly using the current secure k-nearest neighbor strategies over mixed data. Subsequently, in this paper, we build up our past work in [4] and give another response for the PPkNN classifier issue over encoded data. More especially, this paper is not exactly the same as our preliminary work [8] in the going with four viewpoints. In any case, in this paper, we displayed new security primitives, to be particular secure minimum (SMIN), secure slightest out of n numbers (SMINn), secure repeat (SF), and proposed new responses for them. Second, the work in [10] did not give any formal security examination of the fundamental sub-traditions. On the other hand, this paper gives formal security checks of the shrouded sub-traditions and furthermore the PPkNN tradition under the semi-authentic model. In addition, we discuss distinctive methodologies through which the proposed PPkNN tradition can be contacted a tradition that is secure under the threatening setting. Third, our preliminary work in [6] addresses simply secure kNNquery which resembles Stage 1 of PPkNN. Regardless, Stage 2 in PPkNN is totally new. Finally, our observational examinations in Section 6 rely on upon a veritable dataset while the results in [11] rely on upon a reproduced dataset. In addition, new trial results are joined into this paper. 2.3 Threat Model We get the security definitions in the composed work of secure multi-party estimation (SMC) [12], [13], and there are three key antagonistic models under SMC: semi-sensible, incognito and dangerous. In this paper, to make secure and able conventions, we recognize that social events are semi-sensible. Quickly, the running with definition gets the properties of a secured custom under the semi-sensible model [16], [17]. Definition 1: Let ai be the information of get-together $\mathrm{Pi}$, _i(_) be Pi's execution photograph of the custom _ also, bi be the yield for gettogether Pi figured from _. By then,_ is secure if _i(_) can be copied from ai and bi with the true objective that dissipating of the imitated picture is computationally dubious from _i(_). In the above definition, an execution picture general unites the data, the yield and the messages conceded amidst an execution of a convention. To display a custom is secure under semi-true blue model,we for the most part need to demonstrate that the execution photograph of a convention does not release any data concerning the private commitments of taking an interest parties [14]. 2.4 Paillier Cryptosystem The Paillier cryptosystem is an extra substance homomorphic similarly, probabilistic open key encryption organize whose security depends on upon the Decisional Composite Residuosity Assumption [11]. Allow Epk to be as far as possible with open key pk given by $(\mathrm{N}, \mathrm{g})$, where $\mathrm{N}$ is a delayed consequence of two gigantic primes of comparable piece length and $g$ is a generator in Z* N2 . Besides, enable Dsk to sit unbothered as far as possible with confuse key sk. For any given two plaintexts a, b ? ZN, the Paillier encryption organize shows the running with properties: 1) Homomorphic Addition $\operatorname{Dsk}(\operatorname{Epk}(\mathrm{a}+\mathrm{b}))=\operatorname{Dsk}(\operatorname{Epk}(\mathrm{a}) * \operatorname{Epk}(\mathrm{b}) \bmod$ N2); 2) Homomorphic Multiplication $\operatorname{Dsk}(\operatorname{Epk}(\mathrm{a} * \mathrm{~b}))=\operatorname{Dsk}(\operatorname{Epk}(\mathrm{a}) \mathrm{b}$ mod N2); 3) Semantic Security - The encryption plan is semantically secure [14], [17]. Instantly, given a course of action of ciphertexts, an adversary can't find any extra data about the plaintext(s). For compactness, we drop the mod N2 term amidst homomorphic operations in the straggling remains.

\section{PROPOSED SYSTEM}

To reasonably deal with the DMED issue tolerating that the mixed data are outsourced to a cloud. Specifically, we focus on the game plan issue since it is a champion among the most surely understood data mining endeavors. ince each portrayal system has their own specific slack, to be strong, this paper concentrates on executing the $k$ nearest neighbor course of action method over mixed data in the circulated processing condition. The proposed system can execute in any of the application. In the proposed system, understanding prosperity records are protected by covering the delicate data. ata introduction property is proficient here in light of the sort of 
customers. Data anonymization system is used. Data anonymization is somewhat information sanitization i.e. method of ousting the unstable data information

\section{K-NN ALGORITHM}

In case affirmation, the k-Nearest Neighbors count (or k-NN for short) is a non-parametric procedure used for game plan and backslide. $\mathrm{n}$ both cases, the data contains the $\mathrm{k}$ closest planning cases in the segment space. The yield depends on upon whether k-NN is used for portrayal or backslide: (i).In k$\mathrm{NN}$ arrange, the yield is a class enlistment. A challenge is organized by a lion's share vote of its neighbors, with the question being named to the class most customary among its $\mathrm{k}$ nearest neighbors ( $k$ is a positive entire number, ordinarily little). If $k=1$, then the dissent is quite recently delegated to the class of that single nearest neighbor. (ii).In k-NN backslide, the yield is the property estimation for the challenge. This regard is the ordinary of the estimations of its $\mathrm{k}$ nearest neighbors. K-NN is a sort of event based learning, or drowsy acknowledging, where the limit is recently approximated locally and all estimation is yielded until course of action. The kNN count is among the minimum troublesome of all machine learning computations. Both for portrayal and backslide, it can be useful to name weight to the responsibilities of the neighbors, so that the nearer neighbors contribute more to the ordinary than the more far away ones. For example, an average weighting arrangement involves in giving each neighbor a weight of $1 / d$, where $d$ is the division to the neighbor. The neighbors are taken from a course of action of things for which the class (for k-NN gathering) or the question property estimation (for k-NN backslide) is known. This can be considered as the readiness set for the figuring, however no express get ready wander is required. A deficiency of the k-NN figuring is that it is sensitive to the area structure of the data. The computation has nothing to do with and is not to be mixed up for $\mathrm{k}$ infers, another outstanding machine learning technique. The arrangement cases are vectors in a multidimensional segment space, each with a class check. The readiness time of the count involves just of securing the part vectors and class signs of the arrangement tests. In the request arrange, $\mathrm{k}$ is a customer portrayed predictable, and an unlabeled vector (a request or test point) is gathered by consigning the name which is most persistent among the $\mathrm{k}$ planning tests nearest to that question point. A frequently used partition metric for relentless components is Euclidean detachment. For discrete elements, for instance, for substance gathering, another metric can be used, for instance, the cover metric (or Hamming partition). Concerning quality expression microarray data, for example, k-NN has moreover been used with association coefficients, for instance, Pearson and Spearman. As often as possible, the course of action accuracy of k-NN can be upgraded generally if the division metric is discovered with particular counts, for instance, Large Margin Nearest Neighbor or Neighborhood parts examination. A drawback of the essential "larger part voting" game plan happens when the class transport is skewed. That is, instances of a more nonstop class tend to lead the figure of the new case, since they tend to be fundamental among the k-nearest neighbors due to their immense number. One way to deal with vanquish this issue is to quantify the portrayal, considering the division from the test demonstrate each of its $k$ nearest neighbors. The class (or regard, in backslide issues) of each of the $\mathrm{k}$ nearest centers is expanded by a weight comparing to the retrogressive of the partition beginning there to the test point. Another way to deal with thrashing skew is by appearance in data depiction. For example in a self-dealing with guide (SOM), each center is a specialist (a center) of a gathering of similar concentrations, paying little notice to their thickness in the main get ready data. K-NN can then be associated with the SOM. B. Principle Contributions - The issue of insurance sparing packing over mixed data in an outsourced space was kept an eye on similarly starting late [4]. In any case, the present strategy is proposed under a singular customer setting. To the best of our knowledge, there is no present work that addresses the PPODC issue (i.e., under the multi-customer setting). In this paper, we propose a beneficial and novel PPODC tradition that can engage a get-together of customers to outsource their mixed data and what's more the k-infers gathering task absolutely to a joined cloud condition and our own is the vital work along this course. The essential duties of this work are four-cover: - We propose new switches and develop a demand securing Euclidean division work that engages the proposed PPODC tradition to securely choose the data records to the closest packs, a basic walk in each accentuation of the k-infers gathering computation. Furthermore, we propose a novel change for the end condition that engages the PPODC tradition to securely evaluate the end condition over encoded data.

- The proposed game plan satisfies all the alluring properties of PPODC said in the past sub-territory. That is, it secures the protection of each customer's data at all conditions and yields the correct result. Furthermore, once the customer's data is outsourced to the cloud, the customer does not need to participate in any computations of the batching task. We show that the proposed tradition is secure under the standard semireasonable model [1]. Similarly, we speculatively explore the complexities of the proposed tradition. - We display the sensible tangibility of our answer through expansive examinations using a certifiable dataset.

\section{CONCLUSION AND FUTURE WORK}

To guarantee customer security, diverse insurance sparing game plan systems have been proposed over the earlier decade. The present methodology are not important to outsourced database conditions where the data lives fit as a fiddle on an untouchable server. This paper proposed a novel security ensuring k-NN arrange tradition over encoded data in the cloud. ur tradition secures the protection of the data, customer's information request, and covers the data get to plans. We in like manner evaluated the execution of our tradition under different parameter settings. Since upgrading the viability of SMINn is a basic beginning stride for improving the execution of our PPkNN tradition, we plan to look at choice and more beneficial responses for the SMINn issue in our future work. Also, we will analyze and extend our investigation to other course of action figuring's.

\section{REFERENCES}

[1] P. Mell and T. Grance, ?The NIST importance of dispersed registering (draft),? NIST Special Publication, vol. 800, p. 145, 2011.

[2] S. De Capitani di Vimercati, S. Foresti, and P. Samarati, ?Managing and getting to data in the cloud: Privacy risks and approaches,? in Proc. seventh Int. Conf. Risk Security Internet Syst., 2012, pp. 1-9. 1272 IEEE TRANSACTIONS ON KNOWLEDGE AND DATA ENGINEERING, VOL. 27, 
NO. 5, MAY 2015

[3] P. Williams, R. Sion, and B. Carbunar, ?Building châteaux out of mud: Practical get to illustration insurance and rightness on untrusted storage,? in Proc. fifteenth ACM Conf. Comput. Commun. Security, 2008, pp. 139-148.

[4] P. Paillier, ?Public enter cryptosystems in light of composite degree residuosity classes,? in Proc. seventeenth Int. Conf. Theory Appl. Cryptographic Techn., 1999, pp. 223-238.

[5] B. K. Samanthula, Y. Elmehdwi, and W. Jiang, ?k-nearest neighbor arrange over semantically secure encoded social data,? eprint arXiv:1403.5001, 2014. [6] C. Respectability, ?Fully homomorphic encryption using flawless lattices,? in Proc. 41st Annu. ACM Sympos. Speculation Comput., 2009, pp. 169- 178.

[7] C. Respectability and S. Halevi, ?Implementing privileged's totally homomorphic encryption scheme,? in Proc. 30th Annu. Int. Conf. Speculation Appl. Cryptographic Techn.: Adv. Cryptol., 2011, pp. 129-148.

[8] A. Shamir, ?How to share a secret,? Commun. ACM, vol. 22, pp. 612-613, 1979.

[9] D. Bogdanov, S. Laur, and J. Willemson, ?Sharemind: A structure for speedy privacypreserving computations,? in Proc. thirteenth Eur. Symp. Res. Comput. Security: Comput. Security, 2008, pp. 192-206.

[10] R. Agrawal and R. Srikant, ?Privacy-protecting data mining,? ACM Sigmod Rec., vol. 29, pp. 439-450, 2000.

[11] Praveen P., Rama B. (2018) A Novel Approach to Improve the Performance of Divisive Clustering-BST. In: Satapathy S., Bhateja V., Raju K., Janakiramaiah B. (eds) Data Engineering and Intelligent Computing. Advances in Intelligent Systems and Computing, vol 542. Springer, Singapore

[12] P. Praveen, C. J. Babu and B. Rama, "Big data environment for geospatial data analysis," 2016 International Conference on Communication and Electronics Systems (ICCES), Coimbatore, 2016, pp. 1-6. doi: 10.1109/CESYS.2016.7889816

[13] M Sheshikala, D Rajeswara Rao, R Vijaya Prakash, "A MapReduce Framework for Finding Clusters of Colocation Patterns-A Summary of Results" ,Advance Computing Conference (IACC), 2017 IEEE 7th International, Pages 129131

[14] A Novel Rank Based Co-Location Pattern Mining Approach Using Map-Reduce, M Sheshikala, D Rajeswara Rao, R Vijaya Prakash, Journal of Theoretical and Applied Information, Volume 87 Issue 3, Pages 422

[15] Join-Less Approach For Finding Co-Location Patterns-Using Map-Reduce Framework,M Sheshikala, D Rajeswara Rao, R Vijaya Prakash,Journal of Theoretical and Applied Information, Volume 87 Issue 2, Pages 255

[16] M. Sheshikala, D. Rajeswara Rao and R. Vijaya Prakash, Parallel Approach for Finding Co-location Pattern - A Map Reduce Framework, Twelfth International Multi-Conference on Information Processing-2016 (IMCIP-2016)[17] M. Sheshikala, D. Rajeswara Rao and R. Vijaya Prakash, Computation Analysis for Finding Co-Location Patterns using Map-Reduce Framework, Indian Journal of Science and Technology,Vol,10(8),DOI:10.17485/ijst/2017/v10i8/106709, February 2017. 